\title{
A MEMBRANE BREATHER FOR MICRO FUEL CELL WITH HIGH CONCENTRATION METHANOL
}

\author{
De-Sheng Meng, Thomas Cubaud, Chih-Ming Ho, and Chang-Jin "CJ" Kim \\ Mechanical and Aerospace Engineering Department, University of California, Los Angeles \\ Los Angeles, CA 90095, U.S.A.
}

\begin{abstract}
This paper introduces a breather for micro direct methanol fuel cell $(\mu \mathrm{DMFC})$ that removes the gas byproduct $\left(\mathrm{CO}_{2}\right)$ from aqueous methanol fuel. The proposed breather aims to improve the efficiency of a fuel cell by preventing excessive accumulation of gas bubbles in the microchannel where the liquid fuel flows. The release of gas bubbles is visually demonstrated by developing a micro-breather, which integrates microchannels, nano-porous hydrophobic membrane, and an on-chip bubble intake mechanism. The breather achieves successful gas separation from methanol fuel with a concentration as high as $10 \mathrm{M}$ and with pressure tolerance as high as 35psi without leakage, fulfilling the requirement of the next-generation $\mu \mathrm{DMFC}$. This breathing scheme can be useful for other microfluidic applications, where bubbles need to be eliminated from the system.
\end{abstract}

\section{INTRODUCTION}

Due to their potential for high energy capacity and environment-friendly nature, PEM (Proton Exchange Membrane) fuel cells have recently attracted considerable attention as a micro power source, with the $\mu$ DMFCs [1] leading the way.

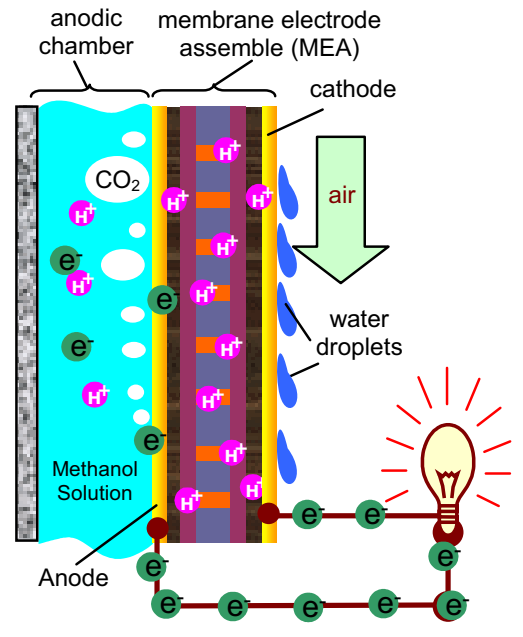

Figure 1. Schematic of DMFCs' fuel stack

In the fuel stack of direct methanol fuel cell as shown by figure 1 , the electrochemical reaction is:

$$
\begin{array}{lr}
\mathrm{CH}_{3} \mathrm{OH}+\mathrm{H}_{2} \mathrm{O} \cdot 6 e^{-}+6 \mathrm{H}^{+}+\mathrm{CO}_{2} \bullet & \text { (anodic side) } \\
1.5 \mathrm{O}_{2}+6 e^{-}+6 \mathrm{H}^{+} \cdot 3 \mathrm{H}_{2} \mathrm{O} & \text { (cathodic side) } \\
\mathrm{CH}_{3} \mathrm{OH}+1.5 \mathrm{O}_{2} \cdot 2 \mathrm{H}_{2} \mathrm{O}+\mathrm{CO}_{2} & \text { (overall reaction) }
\end{array}
$$

According to this reaction, DMFCs generate $\mathrm{CO}_{2}$ gas bubble intrinsically. The small bubbles in large-scale DMFC may not cause much trouble. They are usually carried with the fuel flow to a downstream external gas/liquid separator, essentially an open tank, and get released there. But unique problems occur in their microscale counterparts, where channels may be as small as individual bubbles. As $\mathrm{CO}_{2}$ bubbles fill the microchannel, flow resistance of fuel increases dramatically and the reaction area on PEM surface reduces substantially. Furthermore, pressure buildup by gas accumulation would worsen fuel cross-over of PEM.

It would be most ideal to breathe out these bubbles where they are generated. However, simple openings in the $\mu$ DMFCs are not acceptable as the liquid fuel would leak out through the holes. Meng and Kim [2] have verified that hydrophobic microscale holes release $\mathrm{CO}_{2}$ gas without leakage under moderate pressure. The ability to prevent leakage increases dramatically when the hydrophobic capillaries shrink down to sub-micrometer. In this paper, we report the use of nano-porous hydrophobic membrane for a breather suitable even for high-concentration methanol under high pressures, a challenging condition expected for the nextgeneration fuel cells. In addition to $\mu \mathrm{DMFC}$, the breather's potential applications include microbial (glucose) fuel cell [3] , portable dialysis device [4], water recycling system for space shuttle [5], degasser for HPLC, and micro-mixer [6] .

\section{BREATHING AND LIQUID HOLDING MECHANISM}

Figure 2 represents a simple, idealized model to illustrate how liquid can be held from leaking by its own small meniscus. On the sharp corner of the breathing hole's entrance, this meniscus can assume a range of shapes, so as to balance under varying pressure according to the Laplace-Young equation:

$$
P_{f}-P_{0}=2 \sigma_{f} \cdot \cos (\pi-\alpha) / r
$$

where $P_{f}$ is the pressure of the liquid fuel, $P_{0}$ is the ambient pressure, $\sigma_{f}$ is the surface tension of fuel, $\alpha$ is the angle between meniscus and capillary wall, and $r$ is radius of capillary. Since air bubbles in the fluid are not confined by this kind of interface, they can be breathed out freely.

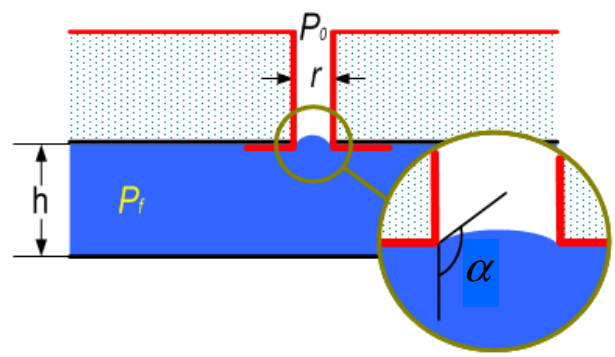

Figure 2. Liquid meniscus in a hydrophobic capillary

The pressure that a hydrophobic capillary can withstand is limited. Given a surface material and a liquid, the maximum contact angle is fixed (known as dynamic advancing contact angle 
or $\theta_{\alpha}$ ). The maximum pressure difference that can be resisted in this breathing hole is:

$$
P_{\max }=\operatorname{Max}\left(P_{f}\right)-P_{0}=2 \sigma_{f} \cdot \cos \left(\pi-\theta_{a}\right) / r
$$

If the fuel pressure is higher than this critical pressure, leakage would happen. Here, we have assumed that the breathing hole is straight and the surface of its wall is smooth. The nanopores in the membrane, however, can be described better by a more realistic model with irregular capillary, as illustrated in figure 3, where the maximum pressure before leakage is defined as:

$$
P_{\max }{ }^{\prime}=2 \sigma_{f} \cdot \operatorname{Max}\left(\cos \left(\pi-\theta_{\text {eff }}\right) / r_{\text {eff }}\right)
$$

with $r_{e f f}$ stands for the effective radius of breathing holes and $\theta_{e f f}$ for the effective advancing contact angle inside the breathing holes. The "Max" implies that the leakage of each breathing hole is defined by its most constricted neck, as shown in figure 3 .

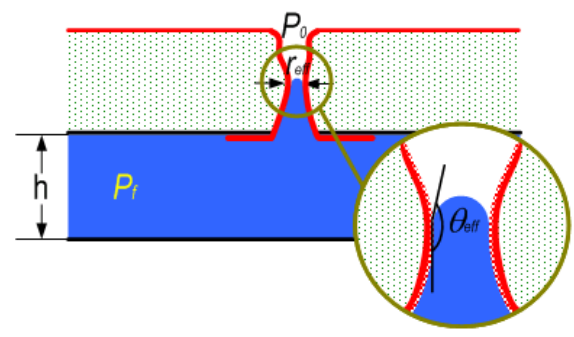

Figure 3. Leakage pressure for irregular capillary

According to equation 3, the irregular breathing holes can usually withstand larger pressure because a rough surface is known to increase the advancing contact angle [7] and the radius of the "neck" can be much smaller than the nominal radius specified by the manufacturer. These two factors tend to increase leakage pressure. Meanwhile, an opposite factor should also be taken into account: given a membrane with many distinct holes on it, the membrane-scale leakage happens on the most susceptible pores. So the leakage pressure of the membrane is:

$$
P_{\text {leak }}=\operatorname{Min}\left(P_{\max }^{\prime}\right)=\operatorname{Min}\left(2 \sigma_{l} \cdot \operatorname{Max}\left(\cos \left(\pi-\theta_{\text {eff }}\right) / r_{\text {eff }}\right)\right)
$$

\section{DEVICE FABRICATION AND TEST}

We have recently demonstrated the removal of chemicalreaction-induced $\mathrm{CO}_{2}$ bubbles from inorganic aqueous solution through Teflon®-coated silicon-micromachined breathing holes (50 $\mu \mathrm{m}$ in diameter)[2]. However, the pressure it could withstand was limited because of the difficulties in the fabrication of microscale breathing holes with uniform hydrophobic coating and mechanically robust structure. Leakage cannot be prevented at all for high-concentration fuels, because the breathing holes are wet much easier by the concentrated fuel with lower surface tension and smaller contact angle. Table 1 summarizes our measurements

\begin{tabular}{|c|c|c|c|c|c|c|}
\hline & & DI water & $1 \mathrm{M}$ & $2 \mathrm{M}$ & $10 \mathrm{M}$ & Methanol \\
\hline \multirow{3}{*}{$\begin{array}{l}\text { Contact angle on } \\
\text { Teflon }{ }^{\text {flat surface }}\end{array}$} & Advancing & $122^{\circ}$ & $119^{\circ}$ & $120^{\circ}$ & $101^{\circ}$ & $67^{\circ}$ \\
\hline & Receding & $105^{\circ}$ & $102^{\circ}$ & $99^{\circ}$ & $60^{\circ}$ & $60^{\circ}$ \\
\hline & Hysteresis & $17^{\circ}$ & $17^{\circ}$ & $21^{\circ}$ & $41^{\circ}$ & $7^{\circ}$ \\
\hline $\begin{array}{l}\text { Surface tension } \\
(\text { dynes } / \mathrm{cm})\end{array}$ & & 72.8 & 66.5 & 57.4 & 39.7 & 22.7 \\
\hline
\end{tabular}
of contact angles with methanol of various concentrations.

Table 1. Interfacial properties of methanol aqueous solution

Moreover, progresses of PEM (Proton Exchange Membrane) technology are expected to substantially reduce the cross-over problem and increase the feasible fuel concentration from current $0.5-2 \mathrm{M}$ to $8-10 \mathrm{M}$ to achieve higher energy efficiency. Challenged by the high concentration fuel, we identify intrinsically hydrophobic porous membranes to construct a breather for high- concentration methanol fuel. This category of membranes has originally been developed for the sample preparation of X-ray spectrochemistry, high performance liquid chromatography, and ultrafiltration. Their $0.1-5 \mu \mathrm{m}$ diameter pores are ideal for the gas breathing application. Advanced membrane technologies have provided diverse membrane materials, including some of the most hydrophobic polymers (e.g. PTFE) known.

Figure 4 shows the scheme to construct a gas permeable microchannel by sandwiched membrane. The breather chip and membrane holder are both fabricated from a same $400 \mu \mathrm{m}$-thick (100) silicon wafer by DRIE etching. A cross-shaped gas-bubble generator [8] is also designed on the breather chip to produce adjustable liquid/gas two-phase flow, corresponding to $\mu \mathrm{DMFC}$ 's fuel flow with $\mathrm{CO}_{2}$ bubbles. Parts of the microchannel on the breather chip are protected by polyimide tape after the DRIE etching has reached the desired depth. After DRIE and subsequent Piranha cleaning, the breather/bubble generator chip is anodically bonded to a piece of Pyrex ${ }^{\circledR}$ glass. Then the membrane is sandwiched between breather chip and membrane holder chip with epoxy to construct a gas-permeable microchannel, analogous to $\mu$ DMFCs' fuel chamber with integrated breather. During the epoxy adhesive bonding, through-holes on both chips are used as alignment marks. The alignment is assisted by strong illumination from below.

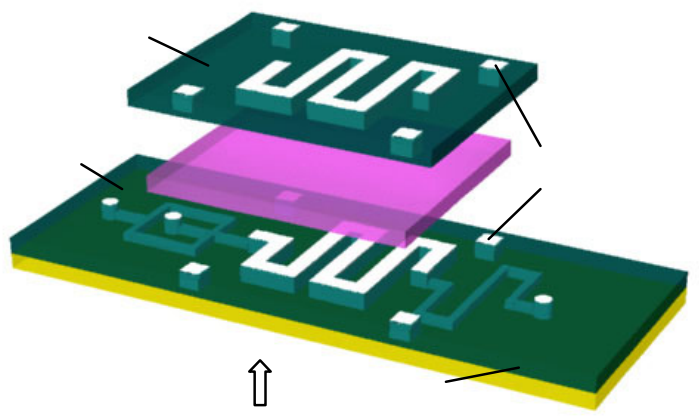

Figure 4. Alignment and bonding setup

The finished breather is shown in figure 5. Gas and liquid are injected via the tubing connected to the breather (not shown in the figure). Pressurized gas is used to inject $\mathrm{CO}_{2}$ directly and water/methanol solution is provided indirectly via a liquid reservoir. Flow meters and pressure sensors are used to monitor the flow rate and pressure inside the microchannel. The experimental data are collected by a computer.

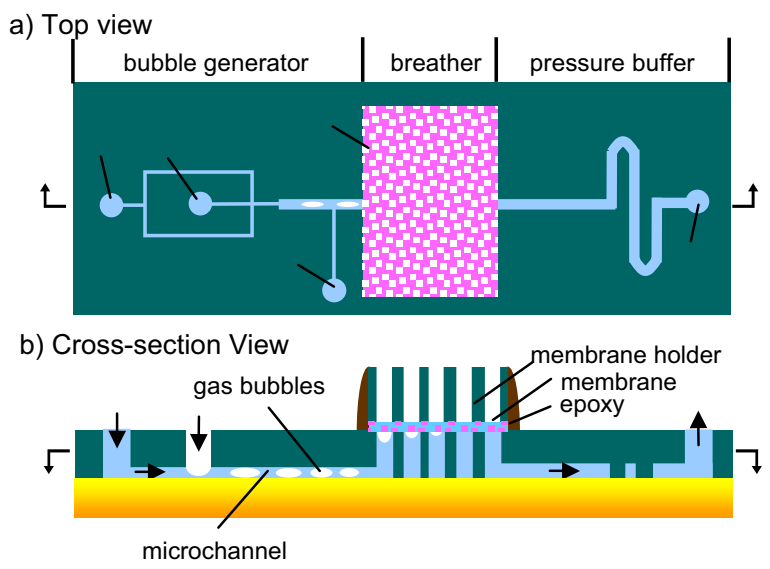

Figure 5. Sandwiched membrane breather 


\section{EXPERIMENTAL RESULTS}

The experiments were carried out with both DI water and $10 \mathrm{M}$ methanol inside membrane breathers of porous PTFE membrane (with $3 \mu \mathrm{m}$ pore in diameter, from Millipore) and porous polypropylene membrane (with $0.2 \mu \mathrm{m}$ pore in diameter, from Chemplex ${ }^{\circledR}$ Industries Inc). During the experiments, the pressure inside the microchannel was controlled to $0.5-2 \mathrm{psi}$. The breathing processes, recorded by a microscope equipped with a CCD camera, were proved to be highly repeatable. The time-series of $0.2 \mu \mathrm{m}$ polypropylene membrane breather are shown in figure 6 . The $3 \mu \mathrm{m}$ PTFE membrane breather demonstrates similar breathing behavior but with a faster breathing rate.

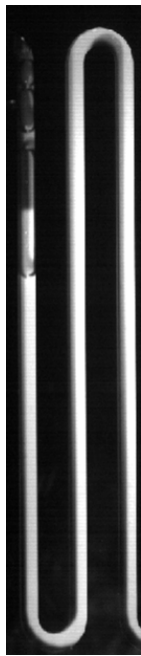

$00 \mathrm{~s}$

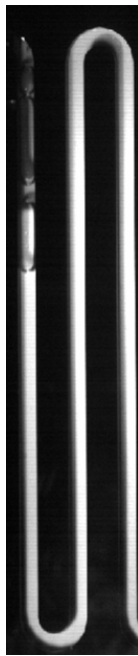

$01 \mathrm{~s}$

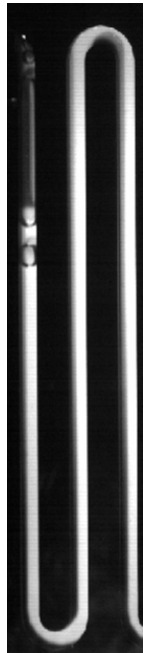

$04 \mathrm{~s}$

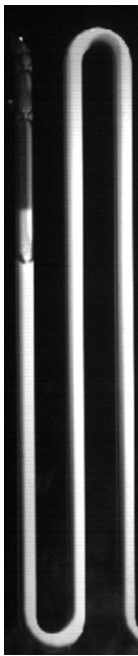

06s

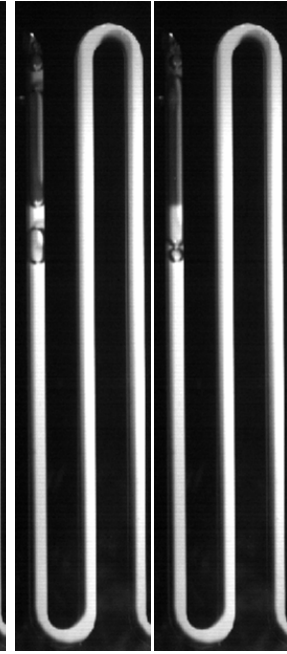

$08 \mathrm{~s}$ 09s
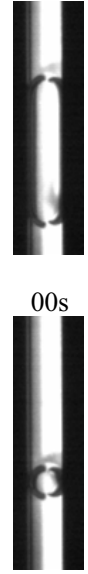

$06 \mathrm{~s}$
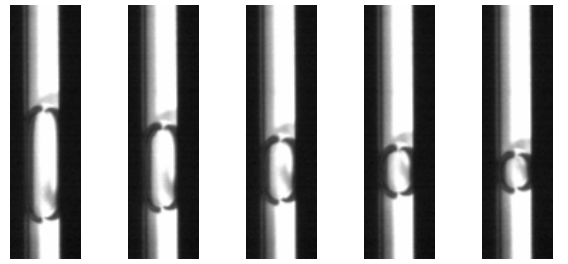

(a) breathing for DI water (no breathing activation)

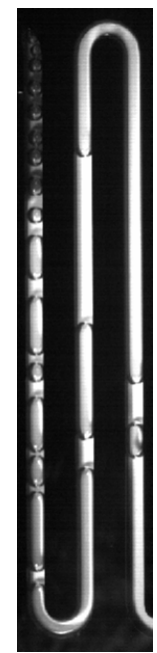

$00 \mathrm{~s}$

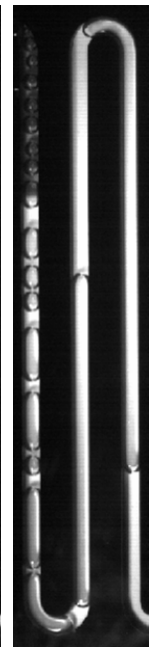

$02 \mathrm{~s}$

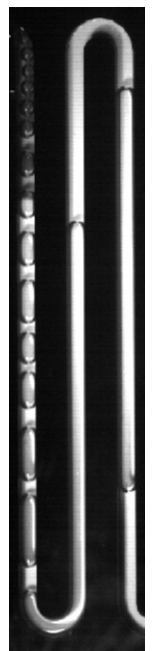

$04 \mathrm{~s}$

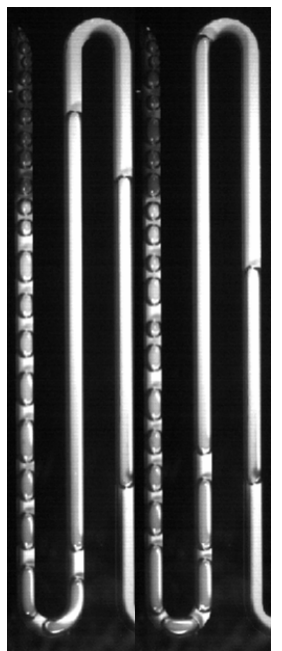

$06 \mathrm{~s}$

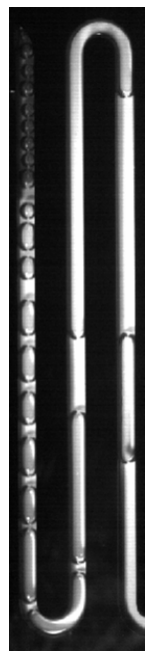

$10 \mathrm{~s}$

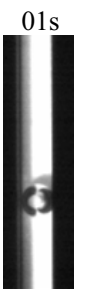

$07 \mathrm{~s}$

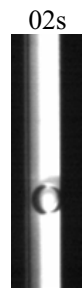

$08 \mathrm{~s}$

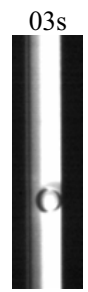

$09 \mathrm{~s}$

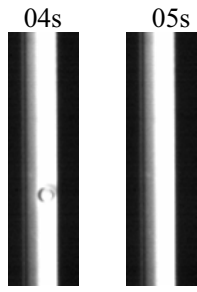

$10 \mathrm{~s}$
$11 \mathrm{~s}$

Figure 6. Breathing process in gas permeable microchannel (transmembrane pressure $=0.8 p s i$ ).

As figure 6(a) shows, the bubbles in DI water are breathed out almost immediately after they touch the membrane. Usually, only the front meniscus is observed to be oscillating within a short distance from the non-permeable part of the microchannel, because breathing and injecting are competing there. Small bubbles can also break from the large one and get breathed out completely.

Figure 6(b) shows similar bubble reduction can also be obtained in high-concentration methanol. However, bubbles in high-concentration methanol tend to stay in the gas permeable microchannel for a longer period than DI water case before they are merged into the largest leading bubble in the very front and then breathed out completely (shown in figure 6(c)). Consequently,

(b) reducing of a single bubble in 10M Methanol

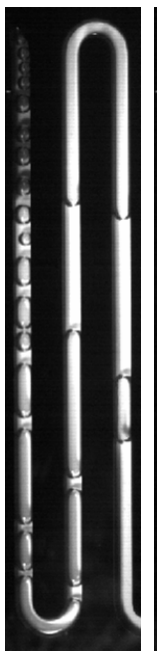

$12 \mathrm{~s}$

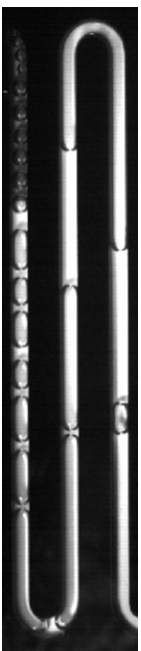

$14 \mathrm{~s}$

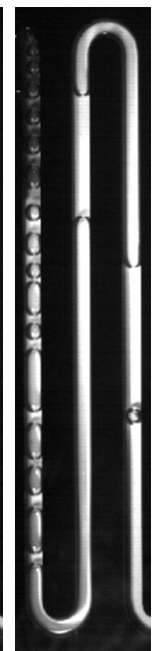

$16 \mathrm{~s}$

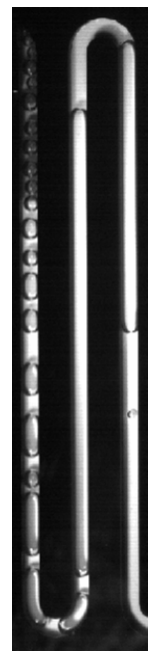

$18 \mathrm{~s}$

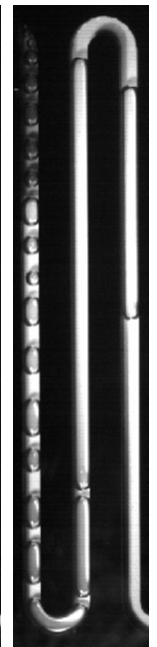

$20 \mathrm{~s}$

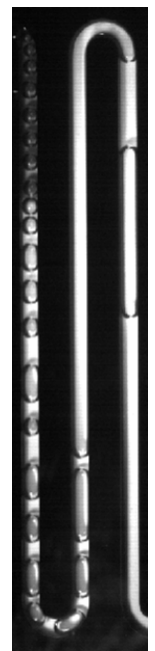

$22 \mathrm{~s}$ a bubble train behind the leading bubble is formed. Since bubbles stay intact for a short period before being breathed out, we state that a "breathing activation" is needed. An explanation is that a tiny liquid droplet can be trapped inside the small capillaries and block the breathing of gas (figure 7). Due to contact angle hysteresis, the contact angle on the head or tail of this droplet ( $\theta_{\text {head }}$ and $\theta_{\text {tail }}$ ) can be different. This introduces a corresponding pressure difference that can hold this droplet in the breathing hole temporarily. However, the eventual success of breathing suggests that the tiny droplet in this hydrophobic nano-pore is unstable. It can finally be removed to clear the breathing hole, or in another word, the breathing can be activated. 


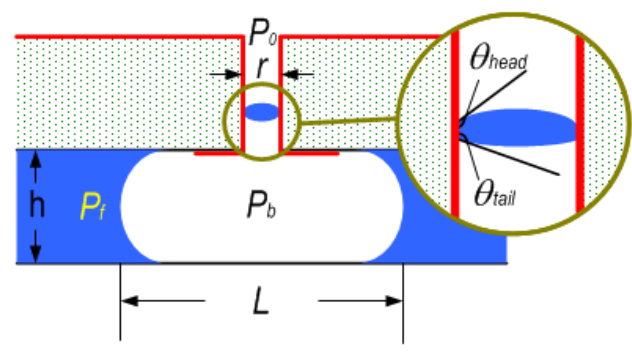

Figure 7. Droplet trapped in a breathing hole

This hypothesis is supported by two facts. First, experiments with DI water, whose contact angle hysteresis is much smaller than that of $10 \mathrm{M}$ methanol (refer to table 1), demonstrate less or no need for breathing activation. Second, the bubble train is observed to be much shorter when the flow rate is reduced. Trapped droplets can be a reasonable explanation, because bubble-covered membrane area gets more opportunities to eliminate the trapped droplets if the flow is slower. Nevertheless, more research is necessary to confirm this hypothesis or find new explanation for the phenomenon of breathing activation. Since the bubble train will evidently increase the flow resistance in the microchannel, theoretical analysis and corresponding design modification should be made in the future to minimize the need for breathing activation in the working $\mu \mathrm{DMFC}$.

The leakage pressure is measured and summarized in table 2 . Since $r_{\text {eff }}$ and $\theta_{\text {eff }}$ are difficult to measure, equation (4) can not be applied directly. The theoretical value is hence calculated from equation (2), using the nominal diameter specified by the manufacturers. Our data shows this estimation of leakage pressure is too conservative for all of the four experiments.

Table 2. Leakage pressure: calculated and measured value

\begin{tabular}{|c|c|c|c|c|c|c|}
\hline & \multicolumn{3}{|c|}{ Porous polypropylene } & \multicolumn{3}{|c|}{ Porous PTFE } \\
\hline & $\begin{array}{l}\text { nominal } \\
\text { diameter }\end{array}$ & $\begin{array}{c}\text { calculated } \\
\text { value }\end{array}$ & $\begin{array}{c}\text { measured } \\
\text { value }\end{array}$ & $\begin{array}{l}\text { nominal } \\
\text { diameter }\end{array}$ & $\begin{array}{c}\text { calculated } \\
\text { value }\end{array}$ & $\begin{array}{c}\text { measured } \\
\text { value }\end{array}$ \\
\hline 10M Methanol & \multirow{2}{*}{$0.2 \mu \mathrm{m}$} & $22 \mathrm{psi}$ & $>35 \mathrm{psi} *$ & \multirow{2}{*}{$3 \mu \mathrm{m}$} & $1.5 \mathrm{psi}$ & $\sim 5 \mathrm{psi}$ \\
\hline DI water & & 110psi & $>35 \mathrm{psi} *$ & & $7.4 \mathrm{psi}$ & $16 \mathrm{psi}$ \\
\hline
\end{tabular}

*membrane breaks at $35 \mathrm{psi}$

The irregular-hole model (as figure 3 shows) is also confirmed by SEM pictures of the two relevant hydrophobic porous membranes, as shown in figure 8 .

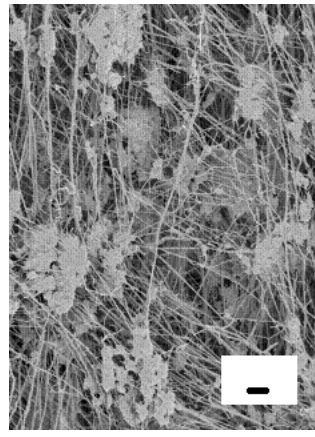

(a) porous polypropylene

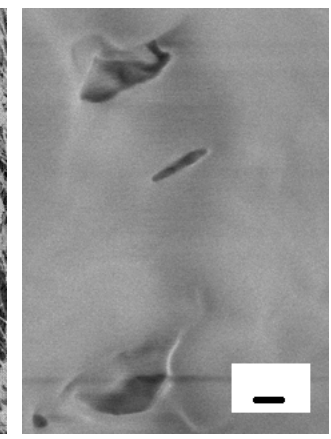

(b) porous PTFE
Figure 8. SEM picture of porous membranes' surface

\section{CONCLUSIONS}

Benefited from micron/submicron hydrophobic breathing holes, the membrane breather achieved $>35$ psi leakage prevention pressure for $10 \mathrm{M}$ methanol and demonstrated stable breathing function in the microchannel. This paves the way for the distributed breather application in $\mu$ DMFC's. The well-established membrane industry provided ample resources for the optimization of this breather to ensure its functionality in $\mu \mathrm{DMFCs}$, as well as other fluidic applications. Further investigation of bubble dynamics, which account for phenomenon like breathing activation, may elucidate the behaviors of microscopic bubbles/droplets inside the breathing holes.

\section{ACKNOWLEDGEMENT}

This work has been supported by DARPA Micro Power Generation Program. The authors wish to thank Professors C. Y. Wang, X. Zhang and X. Zhong, as well as T. J. Yen, G. Q. Lu, Y. $\mathrm{Lu}$ and $\mathrm{H}$. W. Lu for their discussion and help.

\section{REFERENCES}

[1] T. J. Yen, N. Fang, X. Zhang, G. Q. Lu, and C. Y. Wang, "A Micro Methanol Fuel Cell Operating At Near Room Temperature," Applied Physics Letters, vol. 83, pp. 4056-8, 2003.

[2] D.-S. Meng, J. Kim, and C.-J. Kim, "A Distributed Gas Breather for the Micro Direct Methanol Fuel Cell," Proc. IEEE Int. Conf. Micro Electro Mechanical System (MEMS 2003), Kyoto, Japan, 2003, pp. 534-7.

[3] M. Chiao, K. B. Lam, and L. Lin, "Micromachined Microbial Fuel Cells," Proc. IEEE Int. Conf. Micro Electro Mechanical System (MEMS 2003), Kyoto, Japan, 2003, pp. 383-6.

[4] Z. Yang, S. Matsumoto, and R. Maeda, "A prototype of ultrasonic micro-degassing device for portable dialysis system," Sensors and Actuators A, vol. 95, pp. 274-80, 2002.

[5] K. D. Pickering, L. Y. Hammond, and J. L. Garland, "Immobilized Microbe Microgravity Water processing System (IMMWPS) Flight Experiment," Proc. Bioastronautics Investigetors' Workshop, Galveston, Texas, 2001.

[6] A. Guenther, M. Jhunjhunwala, M. A. Schmidt, and K.F.Jensen, "Liquid mixing using inert gas and an integrated gas-liquid separator.," Proc. 7th Int. Conf. Miniaturized Chemical \& BioChemical Analysis Systems ( $\mu-T A S)$, Squaw Valley, CA, 2003, pp. 465-8.

[7] J. Kim and C.-J. Kim, "Nanostructured Surfaces for Dramatic Reduction of Flow Resistance in Droplet-based Microfluidics," Proc. IEEE Int. Conf. Micro Electro Mechanical System (MEMS 2002), Las Vegas, 2002, pp. 479-82.

[8] T. Cubaud and C.-M. Ho, "Transport of Bubbles in Square Microchannels," To Be Published, 2004. 